\title{
Un changement difficile à faire accepter : constats d'une analyse qualitative de l'offre d'aliments sains dans les installations récréatives et sportives
}

Jessie-Lee D. Mclsaac, Ph. D. (1); Sherry L. Jarvis, M.A. (1); Rebecca Spencer, M.A. (2); Sara F. L. Kirk, Ph. D. (1,2)

Diffuser cet article sur Twitter

\section{Résumé}

Les installations récréatives et sportives (IRS) font généralement la promotion de la santé par l'activité physique, mais la qualité de leur environnement alimentaire est souvent négligée. Nous avons exploré les perspectives des intervenants quant aux obstacles à l'offre d'aliments sains dans les IRS en effectuant des entrevues téléphoniques avec dix représentants d'IRS de l'ensemble de la Nouvelle-Écosse. Trois obstacles principaux ont été identifiés : 1) les normes culturelles associées à la nourriture dans les IRS et dans un environnement plus large, 2) la notion ancrée de choix et de responsabilité personnels et 3) les implications financières d'une offre alimentaire saine. Ces obstacles limitent l'offre d'aliments sains dans les IRS et exigent des stratégies variées pour surmonter les normes sociales qui nuisent aux comportements sains.

Mots-clés : culture alimentaire, promotion de la santé, alimentation saine, nutrition, loisirs, sports, politique

\section{Introduction}

Environ les trois quarts des enfants canadiens pratiquent des sports organisés comme le soccer et la natation ${ }^{1}$. Les installations récréatives et sportives (IRS) - un terme qui inclut les centres de conditionnement physique multifonctionnels, les arénas, les patinoires et les terrains de sport - rendent possibles la pratique d'activités physiques structurées et la modélisation d'autres objectifs de promotion de la santé tels que la prévention des blessures ou des espaces sans fumée ${ }^{2}$. Paradoxalement, l'environnement alimentaire dans les IRS contredit souvent les messages de promotion de la santé par son offre en aliments riches en énergie, pauvres en nutriments, rapides à préparer, peu coûteux et lucratifs ${ }^{3-8}$. Cette contradiction apparente constitue une occasion d'améliorer l'environnement alimentaire des IRS pour soutenir les comportements sains des familles qui fréquentent ces endroits ${ }^{9}$.
Les recherches effectuées dans diverses provinces canadiennes ont permis d'identifier les obstacles à une offre alimentaire saine au sein des IRS, notamment les préoccupations financières, la culture alimentaire et la difficulté à convaincre les intervenants de modifier l'offre alimentaire $^{3-6}$. La Nouvelle-Écosse détenant l'un des plus hauts taux de maladies chroniques au Canada ${ }^{10}$, nous avons entrepris de décrire qualitativement les obstacles susceptibles de nuire dans les IRS de la province à l'adoption volontaire des lignes directrices pour une alimentation saine (HERSS Guidelines) ${ }^{11}$ avant leur publication en octobre 2015.

\section{Méthodologie}

Nous avons utilisé une description qualitative pour caractériser les expériences en matière d'offre alimentaire de dix intervenants majeurs d'IRS provinciales (tableau 1).
Points saillants

- Les normes culturelles et les valeurs liées à l'offre alimentaire dans les installations récréatives et sportives risquent de limiter l'influence des interventions élaborées pour encourager une offre alimentaire saine.

- L'idée que leur clientèle n'est pas intéressée et des préoccupations associées à la perte de revenus sont des obstacles persistants chez les intervenants, ce qui est renforcé par la toile de fond sociale qui encourage le choix personnel en tant que déterminant premier de l'offre alimentaire.

- En raison de la complexité des changements de normes sociales, une approche multidimensionnelle est nécessaire pour faire la promotion de choix sains dans les installations récréatives et sportives.

Moins interprétative que d'autres méthodes et demeurant proche des données, ce type de description permet de résumer les évènements dans leur " dimension quotidienne $»^{12}$. Les participants ont été recrutés par courriel au moyen d'un échantillonnage raisonné afin d'identifier les personnes comprenant le mieux les facteurs déterminants de l'offre alimentaire au sein de leur IRS, par exemple les directeurs d'établissement ou les gestionnaires des loisirs ${ }^{13}$. Le comité d'éthique de la recherche de l'Université Dalhousie a approuvé cette étude.

Rattachement des auteures :

1. Healthy Populations Institute, Université Dalhousie, Halifax (Nouvelle-Écosse), Canada

2. School of Health and Human Performance, Université Dalhousie, Halifax (Nouvelle-Écosse), Canada

Correspondance : Sara F. L. Kirk, Healthy Populations Institute, Université Dalhousie, 1318, rue Robie, Halifax (Nouvelle-Écosse) B3H 4R2; tél. : 902-494-8440; courriel : Sara.Kirk@dal.ca 
TABLEAU 1

Caractéristiques des participants

\begin{tabular}{|c|c|c|c|}
\hline $\begin{array}{l}\text { Installation et caractéristiques } \\
\text { des programmes }\end{array}$ & Participants aux entrevues & Caractéristiques du lieu & $\begin{array}{c}\text { Politiques ou lignes directrices sur } \\
\text { I'alimentation saine }\end{array}$ \\
\hline $\begin{array}{l}\text { Centre communautaire multifonc- } \\
\text { tionnel avec aréna aux dimensions de } \\
\text { la LNH, piscine aux dimensions de } \\
\text { compétition, glissade d'eau, centre de } \\
\text { conditionnement physique et mur } \\
\text { d'escalade }\end{array}$ & $\begin{array}{l}\text { Directeur général de l'installation et } \\
\text { gérant des aliments et boissons }\end{array}$ & $\begin{array}{l}\text { Municipalité rurale de plus de } \\
12500 \text { résidents }\end{array}$ & $\begin{array}{l}\text { Politique de l'installation (obliga- } \\
\text { toire) équivalente aux lignes } \\
\text { directrices d'application volontaire } \\
\text { HERSS }\end{array}$ \\
\hline $\begin{array}{l}\text { Programmes et services récréatifs } \\
\text { pour la municipalité, évènements } \\
\text { spéciaux, élaboration de politiques et } \\
\text { supervision des installations locales }\end{array}$ & $\begin{array}{l}\text { Gestionnaire des activités récréatives } \\
\text { municipales }\end{array}$ & $\begin{array}{l}\text { Municipalité rurale fournissant des } \\
\text { services à plus de } 60000 \text { résidents }\end{array}$ & $\begin{array}{l}\text { Aucune politique; lignes directrices } \\
\text { HERSS connues }\end{array}$ \\
\hline $\begin{array}{l}\text { Programmes et services récréatifs } \\
\text { pour la municipalité, évènements } \\
\text { spéciaux, élaboration de politiques et } \\
\text { supervision des installations locales }\end{array}$ & $\begin{array}{l}\text { Gestionnaire des activités récréatives } \\
\text { municipales }\end{array}$ & $\begin{array}{l}\text { Municipalité rurale fournissant des } \\
\text { services à plus de } 8000 \text { résidents }\end{array}$ & $\begin{array}{l}\text { Aucune politique; lignes directrices } \\
\text { municipales équivalentes aux lignes } \\
\text { directrices HERSS }\end{array}$ \\
\hline $\begin{array}{l}\text { Programmes et services récréatifs } \\
\text { pour la municipalité, évènements } \\
\text { spéciaux, élaboration de politiques et } \\
\text { supervision des installations locales }\end{array}$ & $\begin{array}{l}\text { Gestionnaire des activités récréatives } \\
\text { municipales }\end{array}$ & $\begin{array}{l}\text { Municipalité fournissant des services } \\
\text { à plus de } 70000 \text { résidents }\end{array}$ & $\begin{array}{l}\text { Politique municipale (obligatoire) } \\
\text { équivalente aux lignes directrices } \\
\text { HERSS }\end{array}$ \\
\hline $\begin{array}{l}\text { Programmes et services récréatifs } \\
\text { pour la municipalité, évènements } \\
\text { spéciaux, élaboration de politiques et } \\
\text { supervision des installations locales }\end{array}$ & $\begin{array}{l}\text { Gestionnaire des activités récréatives } \\
\text { municipales }\end{array}$ & $\begin{array}{l}\text { Municipalité rurale fournissant des } \\
\text { services à plus de } 25000 \text { résidents }\end{array}$ & $\begin{array}{l}\text { Politique municipale (obligatoire) } \\
\text { équivalente aux lignes directrices } \\
\text { HERSS }\end{array}$ \\
\hline $\begin{array}{l}\text { Programmes et services récréatifs } \\
\text { pour la municipalité, évènements } \\
\text { spéciaux, élaboration de politiques et } \\
\text { supervision des installations locales }\end{array}$ & $\begin{array}{l}\text { Gestionnaire des activités récréatives } \\
\text { municipales }\end{array}$ & $\begin{array}{l}\text { Municipalité rurale de plus de } \\
4700 \text { résidents qui fournit aussi des } \\
\text { services aux communautés } \\
\text { avoisinantes }\end{array}$ & $\begin{array}{l}\text { Aucune politique; lignes directrices } \\
\text { HERSS connues }\end{array}$ \\
\hline $\begin{array}{l}\text { Installation polyvalente pour } \\
\text { réunions, expositions, sports et } \\
\text { divertissement; aréna qui accueille } \\
\text { des évènements spéciaux }\end{array}$ & Directeur général de l'installation & $\begin{array}{l}\text { Municipalité urbaine de plus de } \\
31000 \text { résidents }\end{array}$ & $\begin{array}{l}\text { Aucune politique; lignes directrices } \\
\text { HERSS connues }\end{array}$ \\
\hline $\begin{array}{l}\text { Installation multifonctionnelle avec } \\
\text { arénas, gymnase, salle de condi- } \\
\text { tionnement physique, piscine et salles } \\
\text { de réunion }\end{array}$ & Directeur général de l'installation & $\begin{array}{l}\text { Municipalité rurale desservant plus } \\
\text { de } 50000 \text { résidents, incluant les } \\
\text { communautés avoisinantes }\end{array}$ & $\begin{array}{l}\text { Aucune politique; lignes directrices } \\
\text { HERSS connues }\end{array}$ \\
\hline $\begin{array}{l}\text { Installation multifonctionnelle avec } \\
\text { aréna, centre de santé et de } \\
\text { conditionnement physique, piscines } \\
\text { et salles de réunion, qui accueille des } \\
\text { évènements spéciaux }\end{array}$ & Directeur général de l'installation & $\begin{array}{l}\text { Municipalité urbaine de plus de } \\
67000 \text { résidents }\end{array}$ & $\begin{array}{l}\text { Aucune politique; lignes directrices } \\
\text { HERSS connues }\end{array}$ \\
\hline
\end{tabular}

Abréviations : HERSS, lignes directrices pour une alimentation saine dans les installations récréatives et sportives; LNH, Ligue nationale de hockey.

La collecte de données a reposé sur des entrevues téléphoniques individuelles semistructurées, d'une durée de 45 à 60 minutes et portant sur les expériences des intervenants à propos de l'offre alimentaire en IRS et de leurs perceptions quant à la mise en œuvre de politiques et de pratiques favorisant une alimentation saine. Nous avons recueilli des données entre août et octobre 2014 jusqu'à ce qu'il n'émerge plus de nouvelle information des entrevues. Nous avons utilisé le logiciel d'analyse qualitative (QSR NVivo, version 10) pour la gestion et l'analyse. Nous avons eu recours à un codage ouvert pour sélectionner et définir les codes émergents de manière inductive, suivi d'une approche itérative ${ }^{14,15}$. Deux auteures (Jarvis, Spencer) ont établi les codes, puis les portions d'entrevues ont été codées par chaque auteure pour permettre les comparaisons et toutes les auteures ont contribué au développement des thèmes pour améliorer la crédibilité, l'authenticité et la concordance de ces derniers ${ }^{12,16,17}$

\section{Résultats}

Trois thèmes principaux ont émergé à propos de la mise en œuvre d'une alimentation saine au sein des IRS (citations présentées dans le tableau 2) : 1) les normes culturelles associées aux aliments dans les IRS et dans un environnement plus large, 2) la notion ancrée de choix et de responsabilité personnels et 3 ) les implications financières d'une offre alimentaire saine. Nous présentons ci-dessous un résumé de chaque thème.

\section{Les normes culturelles associées aux aliments dans les IRS et dans un environnement plus large}

Ce thème s'est révélé prédominant et a été présenté comme les normes sociales et les 
TABLEAU 2

Thèmes et citations illustratives

Thème nourriture dans les IRS et dans l'environnement plus largé
Les normes culturelles associées à la

« Le baseball est probablement la plus grande barrière pour le changement nutritionnel à cause de, je ne sais pas, la nostalgie ou la nourriture qui est associée à la culture du baseball, comme les hot-dogs et les chips, les boissons gazeuses, la pizza, les arachides ou les graines de tournesol. »

« Les gens n'embarquaient pas... La majorité des gens qui viennent ici sont des gens du hockey... C'est juste que, c'est la culture qui s'est construite autour des sports... Je veux dire, les joueurs de la LNH, on les voit à la télé faire la promotion de boissons de sport et de chips. »

« ... un gros problème avec les arénas, c'est leurs frites. Ils aiment leurs frites... alors pour moi de devoir leur dire qu'ils ne peuvent plus avoir de frites, et bien, on devrait aussi bien fermer la cantine et l'aréna, un point c'est tout. »

"Tout le monde dit que les célébrations sont importantes, qu'on doit avoir des célébrations, et qu'il doit y avoir de la malbouffe à ces évènements. On dirait qu'il y a vraiment un blocage pour beaucoup de gens, qui ne veulent pas changer leurs habitudes. »

«... le monde du hockey est certainement différent, par exemple, du patinage artistique... En fait, je veux dire, il y a plein de raisons... c'est un sport différent. C'est comme si on comparait la course et, euh, le baseball. C'est une mentalité différente. »

Notion ancrée de choix et de responsabilité personnels

Les implications financières d'une offre alimentaire saine
« Je pense que les gens devraient avoir le choix... c'est ça que je pense. Je ne pense pas que l'aréna soit le bon endroit. Je ne pense pas que nous sommes responsables de décider pour les gens. Je pense que les gens doivent être informés, on doit les éduquer, ils prendront la décision pour moi. »

« L'organisme provincial de chaque sport doit encourager ça chez les organisateurs, les coachs, etc., parce que comme animateur et gérant de l'installation, je ne peux pas tout faire. »

"Je ne pense pas que la majorité des gens qui viennent regarder les matchs de hockey mangent de la poutine tous les soirs à la maison. Alors quand ils viennent ici, c'est leur récompense, OK? Je pense que si je faisais différemment, mes revenus baisseraient. »

«Et le marché va tout dicter... Si le client décide qu'il n'achètera pas de frites et de hot-doǵs, tu ne vendras pas de frites et de hot-dogs. »

« Vendre quelque chose qui va créer un environnement plus sain versus quelque chose qui va te coûter de l'argent ou qui peut t'en faire perdre, ce n'est pas facile... Les gens regardent plus l'argent que la santé, malheureusement. »

«L'année passée, j’ai fait un véritable effort et j'ai engagé un très bon chef... et malgré tout, laisse-moi te dire que ç’a été un échec total. »

Abréviations : IRS, installations récréatives et sportives; LNH, Ligue nationale de hockey.

attentes de la clientèle quant aux types de nourriture à servir dans les IRS. La consommation d'aliments malsains semble être traditionnellement associée à certains sports, comme le hockey et le baseball, avec pour conséquence une résistance au changement en faveur d'options plus saines, par peur que les clients n'achètent pas les nouveaux produits. La conviction que les clients s'attendent à ce que des aliments moins sains soient disponibles lors d'évènements spéciaux a été généralement présentée dans le contexte d'une "gâterie occasionnelle ». À l'inverse, certains types de sport, tels que le patinage artistique, la danse et le soccer, étaient perçus comme plus ouverts à l'idée d'offrir des produits sains.

\section{La notion ancrée de choix et de responsabilité personnels}

Ce thème a été illustré par la tension que les participants ont décrite entre le fait d'encourager le choix personnel par l'offre alimentaire et la responsabilité des IRS d'être des figures de proue de la transformation de l'environnement alimentaire. La plupart des participants estimaient que les employés des IRS ne doivent pas dire aux gens ce qu'ils ont à manger et à ne pas manger. La volonté d'offrir des produits plus sains semblait être acceptable seulement si des aliments moins sains étaient également proposés, le client pouvant ainsi faire son propre choix. Les opinions des participants quant au libre choix se reflétaient dans leur perception de ce que devrait être le rôle des IRS dans la création d'un environnement alimentaire sain : ils n'étaient pas convaincus que les IRS aient pour mandat de modifier l'environnement alimentaire. Certains participants ont suggéré que c'était aux organisations sportives et récréatives provinciales chapeautant les groupes et les installations de sport de diriger cette transition vers une alimentation saine, et non pas à chaque IRS.

\section{Les implications financières d'une offre alimentaire saine}

Ce troisième thème correspond à la crainte exprimée par les participants, surtout par les directeurs d'installations, d'assister à une perte de revenus s'ils vendaient des aliments plus sains ou arrêtaient de vendre 
des aliments moins sains. Dans un contexte culturel où, malgré leur omniprésence, les aliments moins sains sont vus comme des "gâteries ", leur abondance a été attribuée à une demande de la part des clients. Les aliments sains ont été perçus comme plus coûteux, nécessitant plus de préparation et des besoins d'entreposage différents. En outre, le gaspillage des produits invendus s'est révélé une préoccupation particulièrement frappante dans les installations qui n'étaient pas ouvertes chaque jour, comme certains arénas.

\section{Analyse}

Nos constats soulignent les défis rencontrés par les promoteurs de la santé dans la création d'environnements qui encouragent les comportements sains. Laisser les choix personnels définir l'offre alimentaire, comme le privilégient les participants de cette analyse, s'est révélé être un frein à l'amélioration de l'offre d'aliments sains, ce qui est particulièrement préoccupant dans les installations fréquentées par les enfants et les jeunes ${ }^{6}$. Lorsque des aliments sains et malsains sont proposés dans les IRS, les enfants et les jeunes continuent d'acheter principalement les aliments malsains $^{6,18}$. De plus, notre culture actuelle de malbouffe rend la question du libre choix très complexe, parce que plusieurs normes sociales, comportements appris et attentes au sujet des aliments sont très fortement influencés par les campagnes publicitaires de l'industrie alimentaire ${ }^{19,20}$. Les adultes sont tout aussi susceptibles de choisir des aliments malsains ou de vouloir avoir accès dans les IRS à des aliments malsains comme des hot-dogs et des frites ${ }^{21}$, ce qui met en évidence combien l'accès aux aliments malsains dans les IRS est à la fois normalisé et ancré22.

Conformément aux observations d'autres études canadiennes, les participants estimaient que les aliments sains étaient plus chers à acheter, à préparer et à conserver, et moins commercialisables que les aliments malsains ${ }^{2,4,5,23}$. Les données d'une étude d'intervention récente remettent en question cette perception ${ }^{24}$. Dans cette étude, des ressources ont été allouées à un groupe d'intervention afin qu'il change l'environnement alimentaire (nourriture et boissons) dans les clubs sportifs, tandis qu'un groupe témoin ne recevait aucun soutien. Aucune différence significative dans les revenus annuels n'a été observée à la suite de l'intervention entre les clubs du groupe témoin et ceux du groupe expérimental $^{24}$, ce qui signifie que le groupe expérimental n'a pas subi de perte de revenus en raison de la vente de produits plus sains. Des constatations similaires sont ressorties d'une recherche en Alberta $^{23}$. Paradoxalement, cet environnement alimentaire malsain dans les IRS pourrait résulter involontairement d'une meilleure accessibilité à l'activité physique $^{25}$ : certains frais d'utilisation des services offerts en IRS sont financés par les revenus de la restauration, eux-mêmes fortement influencés par les normes sociales des IRS ${ }^{23}$. Pour soutenir les objectifs de promotion de la santé en général, activité physique et saine alimentation ne devraient pas, dans l'idéal, constituer des priorités concurrentes dans les IRS. Cependant, il reste des obstacles à surmonter pour faire évoluer les valeurs liées aux aliments, fortement enracinées.

L'une des forces de notre étude est la participation d'intervenants de différentes régions de la Nouvelle-Écosse et de différents types d'installations, qui étaient porteurs d'expériences variées relativement aux initiatives en alimentation saine. Durant les étapes finales des entrevues et des analyses, aucun nouveau thème n'est ressorti, preuve que nous avions une représentation suffisante des points de vue des intervenants de la province. Cependant, l'une des limites a résidé dans l'utilisation des entrevues téléphoniques : nous avons peut-être laissé passer des indices non verbaux importants qui auraient pu contribuer à l'analyse.

\section{Conclusion}

Nos résultats confirment la nécessité d'agir sur les normes sociales qui font obstacle aux comportements sains, ce qui nécessite la présence d'acteurs en faveur d'une alimentation saine et cherchant à surmonter les résistances aux lignes directrices pour une alimentation saine $e^{5}$. Les stratégies de promotion de la santé susceptibles de faciliter la mise en œuvre de ces lignes directrices sur l'alimentation saine devraient aussi intégrer un soutien social, par exemple de la formation, des ressources et des stratégies qui augmentent la disponibilité et la promotion des aliments sains tout en limitant la commercialisation et la publicité des aliments malsains ${ }^{2,26}$. Des données récentes révèlent que les interventions faisant appel à une combinaison de ces stratégies ont un retentissement plus important sur les choix et les comportements en matière d'alimentation saine ${ }^{17,24}$. Il est clair que, pour promouvoir des options alimentaires saines dans les IRS, une approche multidimensionnelle est essentielle, en raison de la complexité des changements de normes sociales $^{21,27}$. Ainsi, les changements dans les environnements alimentaires dus à la seule volonté d'appliquer des lignes directrices dans les IRS vont se révéler modestes ${ }^{5}$ si l'on n'accorde pas davantage d'attention aux perceptions entourant le coût des aliments sains et aux normes sociales qui continuent de façonner l'offre d'aliments malsains dans ces environnements.

\section{Remerciements}

Les auteures aimeraient remercier tous les participants des installations récréatives et sportives en Nouvelle-Écosse pour leur soutien dans cette recherche.

Cette recherche a reçu l'appui du ministère de la Santé et du Mieux-être de la NouvelleÉcosse, de la Fondation des maladies du cœur et de l'AVC du Canada et des Instituts de recherche en santé du Canada (IRSC, $\mathrm{n}^{\circ}$ de financement 134228). J.-L. McIsaac tient à remercier la Société canadienne du cancer (subvention $n^{\circ}$ 703878). S. Jarvis a reçu une aide salariale de la Fondation des maladies du cœur et de l'AVC du Canada. R. Spencer a reçu du financement dans le cadre d'une bourse de recherche au doctorat des IRSC. S. F. L. Kirk bénéficiait d'une bourse de recherche au doctorat des IRSC durant cette étude.

\section{Conflits d'intérêts}

Les auteures déclarent n'avoir aucun conflit d'intérêts.

\section{Contributions des auteures et avis}

SFLK et JDM ont conçu cette étude. SJ a recueilli les données et $\mathrm{SJ}$ et RS ont fait l'analyse de ces données. Toutes les auteures ont participé à la rédaction du manuscrit et à sa révision critique ainsi qu'à l'interprétation des données. Toutes les auteures ont également lu et approuvé le manuscrit final.

Le contenu de cet article et les opinions qui y sont exprimées n'engagent que les auteurs et ne sont pas forcément représentatifs de la position du Gouvernement du Canada. 


\section{Références}

1. Tremblay MS, Barnes JD, Gonzalez SA, et al. Global Matrix 2.0: Report card grades on the physical activity of children and youth comparing 38 countries. Journal of Physical Activity and Health. 2016;13(11):S343-S366. doi: 10.1123/jpah.2016-0594.

2. Dobbinson SJ, Hayman JA, Livingston PM. Prevalence of health promotion policies in sports clubs in Victoria, Australia. Health Promot Int. 2006; 21(2):121-129. doi: 10.1093/heapro/ dak001.

3. Naylor P-J, Bridgewater L, Purcell M, Ostry A, Wekken SV. Publically Funded Recreation Facilities: Obesogenic Environments for Children and Families? Int $\mathrm{J}$ Environ Res Public Health. 2010;7(5):2208-2221. doi: 10 $.3390 /$ ijerph7052208.

4. Naylor P-J, Vander Wekken S, Trill D, Kirbyson A. Facilitating Healthier Food Environments in Public Recreation Facilities: Results of a Pilot Project in British Columbia, Canada. J Park Recreat Adm. 2010;28(4):37-58.

5. Olstad DL, Lieffers JRL, Raine KD, McCargar LJ. Implementing the Alberta nutrition guidelines for children and youth in a recreational facility. Can J Diet Pract Res. 2011;72(4): 177. doi: 10.3148/72.4.2011.e212.

6. Olstad DL, Raine KD, McCargar LJ. Adopting and implementing nutrition guidelines in recreational facilities: Public and private sector roles. A multiple case study. BMC Public Health. 2012;12:376.

7. Priest N, Armstrong R, Doyle J, Waters E. Policy interventions implemented through sporting organisations for promoting healthy behaviour change [Internet]. Dans : The Cochrane Collaboration (dir.), Cochrane Database of Systematic Reviews. Chichester. UK: John Wiley and Sons; 2008. doi: 10.1002/14651858.CD004809.pub3.

8. Thomas HM, Irwin JD. Food choices in recreation facilities: operators' and patrons' perspectives. Can J Diet Pract Res Publ Dietit Can. 2010;71(4):180185. doi: 10.3148/71.4.2010.180.
9. World Cancer Research Fund International. NOURISHING framework [Internet]. En ligne à : http://www.wcrf.org /int/policy/nourishing-framework

10. Province of Nova Scotia. Thrive! A plan for a healthier Nova Scotia [Internet]. En ligne à : https://thrive. novascotia.ca/

11. Province of Nova Scotia. Healthy eating in recreation and sport settings guidelines [Internet]. En ligne à : https://thrive.novascotia.ca/node $/ 8311$

12. Neergaard MA, Olesen F, Andersen RS, Sondergaard J. Qualitative description - the poor cousin of health research? BMC Med Res Methodol. 2009;16;9(1):52. doi: 10.1186/1471 $-2288-9-52$.

13. Sandelowski M. Whatever happened to qualitative description? Res Nurs Health. 2000;23(4):334-340. doi: 10.1002 /1098-240X(200008)23:4\%3C334::AID -NUR9\%3E3.0.CO;2-G.

14. Patton MQ. Qualitative Research and Evaluation Methods. 3rd ed. Thousand Oaks, CA : Sage Publications; 2002.

15. Miles MB, Huberman AM. Qualitative Data Analysis: An expanded sourcebook. 2nd ed. Thousand Oaks, CA: Sage Publications; 1994.

16. Milne J, Oberle K. Enhancing rigor in qualitative description: a case study. J Wound Ostomy Cont Nurs Off Publ Wound Ostomy Cont Nurses Soc WOCN. 2005 Dec;32(6):413-420. doi: 10.1097/00152192-200511000-00014.

17. Morrow SL. Quality and trustworthiness in qualitative research in counseling psychology. J Couns Psychol. 2005;52(2):250-60. doi: 10.1037/0022 -0167.52 .2 .250 .

18. Van Hulst A, Barnett TA, Déry V, Côté G, Colin C. Health-promoting vending machines: evaluation of a pediatric hospital intervention. Can J Diet Pract Res Publ Dietit Can. 2013;74(1):28-34. doi: 10.3148/74.1.2013.28.

19. Elliott C. "It's junk food and chicken nuggets": Children's perspectives on "kids" food' and the question of food classification. J Consum Behav. 2011 May 1;10(3):133-40.
20. Ludwig DS, Nestle M. Can the food industry play a constructive role in the obesity epidemic? JAMA. doi: 10.1001 /jama.300.15.1808.

21. Olstad DL, Goonewardene LA, McCargar LJ, Raine KD. Choosing healthier foods in recreational sports settings: a mixed methods investigation of the impact of nudging and an economic incentive. Int J Behav Nutr Phys Act. 2014;11:6. doi: 10.1111/j.1468 $-0009.2009 .00550 . x$.

22. Sallis JF, Glanz K. Physical activity and food environments: solutions to the obesity epidemic. Milbank Q. 2009 Mar;87(1):123-54. doi: 10.1111/j.1468 $-0009.2009 .00550 . x$.

23. Olstad DL, Downs SM, Raine KD, Berry TR, McCargar LJ. Improving children's nutrition environments: a survey of adoption and implementation of nutrition guidelines in recreational facilities. BMC Public Health. 2011;11:423. doi: 10.1186/1471-2458-11 $-423$.

24. Wolfenden L, Kingsland M, Rowland BC, Dodds P, Gillham K, Yoong SL, et al. Improving availability, promotion and purchase of fruit and vegetable and non sugar-sweetened drink products at community sporting clubs: a randomised trial. Int $\mathrm{J}$ Behav Nutr Phys Act. 2015;12:35. doi: 10.1186 /s12966-015-0193-5.

25. Olstad DL, Raine KD. Profit versus public health: the need to improve the food environment in recreational facilities. Can J Public Health Rev. 2013; 104(2):e167-e169.

26. Social Research and Demonstration Corporation. Evaluation of the Healthy Food and Beverage Sales in Recreation Facilities and Local Government Buildings Initiative [Internet]. Victoria, BC : University of Victoria; 2010. En ligne à : http://www.srdc.org/uploads /BCHLA_HealthyFood.pdf

27. Chan RSM, Woo J. Prevention of overweight and obesity: how effective is the current public health approach. Int J Environ Res Public Health. 2010;7(3): 765-783. 